\title{
El rol laboral de la mujer en la génesis del Estado totalitario español (1938-1945): una contribución a su estudio
}

\author{
CARMEN GÓMEZ GÓMEZ Y \\ JULIO PEREZ SERRANO \\ Universidad de Cádiz
}

\section{INTRODUCCIÓN}

El cambio de régimen político que se inició en España con el denominado "Alzamiento Nacional» y que se consolidó posteriormente tras el triunfo de los "nacionales" en la Guerra Civil supuso, no cabe duda, una tremenda convulsión de incuestionables efectos sobre el conjunto de la sociedad española. De todos los colectivos quizás fuese el femenino el que se vio afectado más negativamente, y en concreto, las mujeres pertenecientes a los sectores populares, que hubieron de sufrir con la máxima intensidad la presión ideológica del nuevo Régimen y la marginación política, social y económica. Porque, si bien en los países capitalistas de Europa Occidental y en los EE.UU. (y con anterioridad en la Unión Soviética), sobre todo a partir de 1945 , se iban a generar condiciones objetivas y subjetivas claramente favorables para la plena integración de la mujer en el proceso productivo, en nuestro país se pondría en funcionamiento en la misma época una pesada maquinaria superestructural que acabaría sumiéndola en una situación -aún persistente en buena medida- de estancamiento social y marginación económica.

La Revista de Sanidad e Higiene Públicas entre los años 1938 y 1945 constituye, en este sentido, un documento de extraordinario valor, puesto que, a través de ella, podemos conocer con bastante aproximación cuáles eran las bases teóricas sobre las que los diseñadores del Estado totalitario español pretendían sustentar su idea de lo que debería ser el papel de la mujer en el nuevo orden social. En las páginas de esta revista se reflejan con asombrosa nitidez cuá- 
les eran sus duras condiciones de vida y de trabajo, sus enormes carencias culturales, la falta de asistencia social que sufrían tanto ellas como sus hijos y, en definitiva, la compleja problemática que este sector ofrecía a ojos de los responsables del naciente Estado fascista.

Este boletín de divulgación sanitaria para médicos y profesionales de la higiene (1) es valioso por diversas razones. En primer lugar, porque desde él se teoriza, se da homogeneidad y se orienta la reconstrucción de la Sanidad pública de acuerdo a las líneas programáticas e ideológicas del gobierno fascista. En segundo lugar, porque las directrices y propuestas recogidas en la publicación -la mayor parte de ellas todavía en germen - van destinadas a abordar la situación sanitaria de los sectores más deprimidos del cuerpo social, asistidos hasta entonces con un carácter eminentemente benéfico. En tercer lugar, porque, en consecuencia, la atención se centrará durante estos primeros años del Régimen en las mujeres de la clase obrera y demás sectores populares, para las cuales se conforma un modelo sanitario orientado básicamente a proteger y fomentar la actividad reproductora, considerada ahora como la principal función social de la mujer. Finalmente, porque al exigir las cuestiones referentes a la Salud Pública un tratamiento interdisciplinar - más aún cuando se hace de ellas un estudio que incorpora la perspectiva histórica- nos situamos ante la ocasión de poner en contacto la Historia, ciencia eminentemente interdisciplinaria, y la Medicina en su vertiente social, tal vez la más "comprometida".

\section{EL PAPEL DE LA MUJER EN EL FASCISMO ESPAÑOL}

Ya hemos dicho que en la RSHP aparecen delineados los ejes fundamentales de la función social de la mujer para el fascismo. De un lado, una política demográfica abiertamente populacionista, y de otro, la instauración de una sociedad articulada sobre la base de la unidad familiar, donde se fortalece física, moral e ideológicamente a los hijos. Es decir, dos serían las tareas principales de la mujer para el fascismo: la reproducción de los efectivos po-

(1) Cfr. VAlLejo DE SIMÓN, A.: «Reorganización de los Servicios Sanitarios en la Zona liberaday. Revista de Sanidad e Higiene Públicas (en adelante RSHP), I, 1-12 (1938-1939), p. 24. 
blacionales y la educación-adoctrinamiento de los hijos en el ámbito familiar.

El crecimiento demográfico constituye, en efecto, una obsesión no sólo para el fascismo español, sino para el conjunto de este movimiento a nivel internacional. Así lo manifiestan los profesionales de la medicina cuando proponen para España medidas análogas a las observadas, a menudo directamente, por ellos en Italia o Alemania (2). No es de extrañar, pues, que la mortalidad materno-infantil sea la temática predominante en estos cinco primeros años de la publicación, dadas las elevadas tasas de mortalidad y la lacra constituida por la acción de las enfermedades infectocontagiosas, la mala nutrición y las precarias condiciones higiénicas que caracterizan a nuestra postguerra. Desde esta perspectiva será desde donde la mujer se asome más frecuentemente a las páginas de la revista. En palabras de L. Cortejoso "[...] en esta santa defensa de la infancia está la base fundamental de la política del Fascismo, que atiende no sólo a la calidad, sino a la cantidad de los descendientes" (3). De ahí las furibundas y repetidas condenas del aborto y de todo tipo de prácticas contraceptivas, exponente de las cuales pueden ser los trabajos de F. Murillo y de J.L. Morales y González fechados en 1941 y 1942 respectivamente (4). También en la misma línea de defensa de esta política demográfica, J. Vinagre reconocerá que "la consigna dada por el Caudillo, de aumentar [...] el número de habitantes a 40 millones para alcanzar el rango histórico [...] nos hizo pensar sobre cuáles serían las normas [...] para poder cumplir y satisfacer los deseos de nuestro Caudillo" (5). La responsabilidad de hacer posible este extraordinario despegue demográfico habría de recaer, no obstante, según los ideólogos del Régimen, sobre las mujeres de la clase obrera, que de esta forma habrían de verse mayoritariamente relegadas de la producción y vinculadas inexorablemente a las tareas del hogar; como nos dice $\mathrm{E}$. Alvarez, la misión reproductora debería recaer

(2) Vid. CORTEjOSO, L.: "La defensa de la infancia en Italia». RSHP, I, 1-12 (1938-1939), pp. 807-812; MURILLO, F.: "Política de repoblación». RSHP (1941), pp. 209-225.

(3) CORTEJOSO, L.: "La defensa de la infancia..", art. cit., p. 807.

(4) Vid. MURILLO, F.: "Política de repoblación...", art. cit. pp. 209 ss.; MORALES Y GONZÁLEZ, J.L.: "Programa totalitario de Sanidad y Asistencia social infantil». RSHP, XVI, 4 (1942), p. 313.

(5) VINAGRE, J.: «Mortalidad materna y fetal en Badajoz y su provincia, comparada con la de España durante el decenio 1926-1935\%. RSHP, XVII, 1 (1943), p. 70. 
sobre «la clase mejor, la verdadera proletaria, puesto que es la que, s1 no tiene más hijos, al menos cría más y llega a dar a la Patria más soldados $[\ldots]$ " (6).

Por lo que se refiere a su función educadora, la mujer como madre es la encargada de transmitir a los hijos los valores del nacionalsindicalismo y de la religión católica, dentro, claro está, del ámbito familiar. El propio E. Alvarez hará mención expresa de ello cuando afirme, refiriéndose al ideario fascista, que «luchamos por la Religión y por la Patria, y [...] no concebimos que una madre no sepa y, por tanto, no pueda enseñar el catecismo a sus hijos [...] (7). Ya desde niña se ha de preparar a la mujer para que desarrolle en el futuro este papel decisivo en la transmisión de la ideología dominante y el cuidado general de los hijos. Se propone impartir enseñanza puericultora desde los cursos superiores de la escuela, enseñanza que se haría extensiva a la "Normal de Maestras" (8). Otro ejemplo de ello lo tenemos en el proyecto de I. Hernández para crear, adscrita a la Escuela Maternal, una "sección maternal" en la que se impartieran enseñanzas prácticas sobre el cuidado y la formación integral de los hijos; estas secciones, aunque estarían dedicadas al conjunto de la población femenina adulta, contarían también con la asistencia de las jóvenes alumnas (12 años) de las escuelas públicas (9). En 1938 en la mayor parte de las "provincias conquistadas" se habían organizado ya cursillos de puericultura exclusivamente para mujeres, donde, a juzgar por los programas que conocemos, muy probablemente junto a nociones médicas fuesen transmitidos también los principales referentes ideológicos del nuevo Estado (10).

A la mujer que trabaja fuera del hogar se le dedica, sin embargo, una escasa atención, como prueba el hecho de que en cinco años de publicación sólo un artículo haga referencia específicamente a ella, e incluso en este caso la temática abordada, la lactancia, está en íntima relación con la mencionada función reproductora

(6) ALVAREZ ROMERO, E.: "Tuberculosis: vivienda, higiene infantil». RSHP, I, 1-12 (1938-1939), p. 384.

(7) Ibídem, p. 381.

(8) Ibídem, p. 383.

(9) Cfr. HERNÁNDEZ, 1.: "La asistencia social al niño en Tenerife. Guardería infantil». RSHP, I, 1-12 (1938-1939), pp. 200-205.

(10) Cfr. BALEN, J.: «Breve Noticiario de los Servicios de Higiene infantil». RSHP, I. 1-12 (1938-1939), pp. 155-160. 
que el Régimen le asigna (11). No obstante, hay que matizar que el tratamiento que se da a la mujer como "trabajadora" difiere sensiblemente según provenga de los estudios realizados por los higienistas de la infancia o los higienistas del trabajo. Estos últimos (Dantín Gallego a la cabeza) propugnan mejoras legales, aportan proyectos para combatir las enfermedades y la mortalidad profesionales y elaboran estadísticas. La mujer es estudiada en este contexto como lo que realmente es, un elemento marginal en la producción, sin que en ningún momento se proponga tampoco su integración en el mundo laboral. A lo largo de toda la revista vamos asistiendo, asimismo, a la progresiva consolidación de la división de funciones en el trabajo por razón del sexo, quedando reducida la actividad femenina casi por completo precisamente al ámbito de la Sanidad y a la Asistencia Social (12).

\section{LAS CONDICIONES DE VIDA DE LA MUJER EN LA POSTGUERRA}

Por medio de la lectura de la revista podemos ponernos en contacto con las duras realidades de nuestra postguerra. Ya hemos señalado cómo las condiciones de vida eran especialmente adversas para el colectivo femenino, siendo esta situación todavía más precaria en el caso de las zonas rurales, donde se asienta casi el $80 \%$ de la población.

En lo económico, la revista nos presenta a una mujer que acude — cuando lo hace- a los Dispensarios de los Servicios de Higiene determinada por la escasez de recursos y por la responsabilidad de una numerosa prole. A menudo reside en zonas carentes de infraestructura urbanística (sin agua corriente, alcantarillado, etc.), con evidente hacinamiento de los miembros de la familia, que les lleva a convivir incluso con sus propios animales domésticos. En sus grandes líneas este cuadro es el que nos presenta, por ejemplo, J.D. Prada al describirnos la situación de Macotera, población agrícola de Salamanca, en 1938-39 (13).

(11) Cfr. ITURRIAGA, E. de: “El trabajo y la mujer. Las salas de lactancia en fábricas y talleres". RSHP (1941), pp. 390-396.

(12) Entre otros, Vid. TUREGANO, J.F.; DANTÍN GALLEGO, J.; BENÍTEZ RAMÍREZ, A.: “Selección médico psicotécnica para profesiones sanitarias». RSHP (1945), pp. 303-326.

(13) Cfr. PRADA, J.D.: "Nota preliminar sobre mortalidad infantil de Salamanca». RSHP, I, 1-12 (1938-1939), pp. 186-193. 
En lo referente al nivel cultural, las mujeres españolas manifiestan un alto grado de analfabetismo. En el caso de Córdoba, un $48,89 \%$ de las madres que acuden al Servicio de Higiene Infantil en el año 1942 son completamente analfabetas (14). A juicio de los higienistas del Régimen, esta falta de capacitación cultural estará en el origen de la errónea atención que las madres prestan a sus hijos y al cuidado de su propia maternidad, desoyendo las continuas llamadas emitidas desde las instancias públicas para que acudan a los organismos asistenciales dispuestos por el nuevo Estado. A esta circunstancia más que a la situación económica precaria -y en esto hacen hincapié repetidas veces los autores- atribuyen el alto índice de mortalidad materno-infantil. Según un estudio realizado por C. Irízar en Navarra, alrededor del $50 \%$ de estas muertes entre los años 1930 y 1935 podrían haberse evitado con una correcta prevención y educación sanitaria (15).

En el plano sanitario, la situación de la mujer no es más favorable, tanto en lo relativo a la maternidad como en el ámbito del trabajo. Es corriente que carezca de una adecuada asistencia durante el embarazo, el parto y el puerperio, lo que hace que exista un alto índice de abortos espontáneos, de mortalidad materno-infantil y de taras congénitas en el feto. Durante el decenio de 1926 a 1935, se constata que de cada 333 partos acaecidos en Badajoz muere una mujer; a juicio del autor, hubiera sido posible evitar una buena parte de las muertes de haber existido una adecuada vigilancia prenatal y una mejora en los servicios prestados en los paritorios (16). Otros autores introducen como factor causal de esta elevada mortalidad, el hecho de que durante el embarazo fuese frecuente que la mujer continuase desarrollando labores pesadas en el campo o en la industria (17), sin la suficiente protección legislativa laboral; el propio L. Pedraza Carrasco pide, desde las páginas de la revista, en 1940 que se extienda la Ley del Seguro de Maternidad (sólo vigente para obreras industriales) a obreras agríco-

(14) Cfr. BAUTISTA TIRADO, J.: «Balance de un año de labor puericultora». RSHP, XVII, 5 (1943), pp. 529-535.

(15) Cfr. IRİZAR, C.: "Memoria-resumen del Servicio de Higiene infantil de Navarra durante los años 1934-1935». RSHP, 1, 1-12 (1938-1939), pp. 155-160.

(16) Cfr. VINAGRE, J.: "Mortalidad materna y fetal...» art. cit., pp. 70 ss.; IDEM: "Organización de la lucha contra la mortalidad materna y fetalı. RSHP, XVII, 4 (1943), pp. 429-437.

(17) Cfr. PRADA, J.D.: "Nota preliminar sobre mortalidad...", art. cit., pp. 186 ss. 
las y mujeres de obreros (18). Una muestra más de las malas condiciones de vida de las mujeres de nuestra postguerra la constituye, asimismo, la gran propagación que las enfermedades venéreas alcanzaron sobre ellas; un estudio referido a Córdoba recoge que en 1939, de 530 embarazadas vistas en las consultas de Higiene Prenatal de aquella provincia, un 15,6\% estaban afectadas por la sífilis (19), y esta situación parece que continuaba aún sin resolverse en 1943, como demuestra J. López Barroso (20).

\section{EL MARCO JURÍDICO DEL TRABAJO FEMENINO}

De todo lo dicho se deriva que un primer rasgo diferencial del colectivo femenino en estos años fue precisamente su escasa incorporación al mundo del trabajo. Aunque el trabajo de la mujer estuviera protegido y contemplado como un derecho en el ámbito jurídico, lo cierto es que ni se hallaba suficientemente concretado en la legislación, ni en la práctica era extensivo a la mayoría de las mujeres. Por el contrario, las mismas leyes apuntan hacia una progresiva desvinculación de la mujer del ámbito fabril, fomentando el trabajo a domicilio y realizando una acérrima defensa de la maternidad, como se refleja claramente en el Fuero del Trabajo (21).

Para conocer con detalle la evolución de la legislación relativa al trabajo femenino contamos con un extenso artículo de excepcional interés, el de J. Dantín Gallego titulado "Antecedentes para el estudio de la Higiene industrial en España" (22). Por él sabemos que desde 1873 hasta 1940 se suceden diversas leyes y disposiciones que regulan el trabajo de la mujer, vinculándolo casi siempre al de los menores. Entre 1873 y 1923 queda configurada la Ley del Trabajo de las mujeres y los niños. En 1897 se legisla sobre el trabajo de las mujeres y los menores en las minas; en 1908 aparece

(18) Cfr. PEDRAZA CARRASCO, L.: «Nota previa para un estudio nacional de las causas de mortalidad feto-infantil». RSHP, XIV, 1-6 (1940), pp. 18-25.

(19) Cfr. PÉREZ MATA, J.: "Importancia de la profilaxis sifilítica en las consultas de Higiene prenatalı. RSHP, 1, 1-12 (1938-1939), pp. 458 ss.

(20) Cfr. LÓPEZ BARROSO, J.: «El Centro Secundario de Higiene de Santoña. Resumen de los servicios prestados hasta fines de 1941». RSHP, XVII, 2 (1943), pp. 190 ss.

(21) Vid. MORALES Y GONZÁLEZ, J.L.: "Programa totalitario de Sanidad...", art. cit., pp. 304 ss.

(22) DAN'TíN GALLEGO, J.: "Antecedentes para el estudio de la Higiene industrial en Españan. RSHP, XIV, 1-6 (1940), pp. 353-426. 
un decreto clasificando las industrias prohibidas a mujeres y niños; en 1920 se prohíbe el trabajo nocturno de la mujer. Ya en plena dictadura de Primo de Rivera se reincide sobre esta cuestión en el Decreto-ley sobre el descanso nocturno de la mujer obrera (1927). Sin embargo, habremos de esperar hasta 1931 para asistir a la implantación del Seguro Obligatorio de Maternidad, del que ya indicamos que aún en 1941 no se hallaba extendido a toda la población activa femenina.

Los legisladores fascistas consideraron siempre insuficientes estas medidas y, al menos formalmente, pretendieron ampliar y hacer más efectiva la protección al trabajo femenino, aunque en la práctica lo que hicieron fue crear las condiciones para la progresiva desvinculación de la mujer del ámbito fabril. El propio Dantín Gallego celebra que en el Fuero del Trabajo, promulgado en marzo de 19.38 , se regule el trabajo a domicilio, se prohíba el trabajo nocturno de mujeres y niños y, como se reconoce abiertamente, se intente "[libertar] a la mujer casada del taller y de la fábrica» (artículo II.1), a la vez que se subraya su papel reproductor por medio de la extensión de los seguros sociales, entre ellos el de Maternidad (artículo X.2) (23). Poco después, el Código del Trabajo volverá a centrar su atención sobre el trabajo femenino, ofreciendo a la mujer una especial protección en lo concerniente a accidentes laborales (24).

El avance de la política laboral del fascismo exigiría, una vez terminada la contienda civil, el desarrollo de una Ley de Sanidad del Trabajo, que se encontraba ya en germen en 1942, inspirada en la legislación de 1900. Así lo vieron también los higienistas de la RSHP, y concretamente Dantín Gallego, quien en ese mismo año realizará desde las páginas de la revista una propuesta de bases para sacar adelante la misma; la base $10 .{ }^{a}$ y última estará dedicada precisamente al trabajo femenino, vinculándolo al paro obrero, e insistiendo de nuevo en que sea realizado a domicilio (25).

En la misma línea de defensa de la maternidad, en $1941 \mathrm{E}$. Iturriaga pediría que las empresas españolas se acogiesen al Convenio de Washington -firmado en 1919 y al cual en 1941 se habían adherido ya otros 48 países-, por el que, entre otros objetivos, se pretenderían regular la jornada laboral femenina y las cues-

(23) Cfr. lbidem, passim.

(24) En las disposiciones 9." y 10. ${ }^{a}$ del artículo 246.

(25) Cfr. DANTÍN GALLEGO, J.: "El concepto legal y administrativo de la Sanidad cn el trabajo". RSHP, XVI, 1-6 (1942), pp. 232 ss. 
tiones relativas a la maternidad en la mujer trabajadora (26). El autor valora particularmente la implantación de las denominadas «Salas de Lactancia», con el fin de disminuir la mortalidad infantil. No obstante, éstas sólo habían logrado ponerse en funcionamiento en un $29 \%$ de los países adheridos. Y es comprensible que esta medida no se hubiese extendido con mayor rapidez - y que tampoco llegara a ponerse en práctica finalmente en España-, puesto que exigía costosas instalaciones, personal altamente cualificado y, al permitir que la mujer se ausentara en pausas de media hora para amamantar a sus hijos, disminuía sensiblemente la productividad de las obreras.

\section{LA PRESENCIA DE LA MUJER EN LOS SECTORES PRODUCTIVOS}

Sobre la participación de la mujer en los diversos sectores productivos entre 1938 y 1945 encontramos algunas referencias en la RSHP. Por ellas es posible reconstruir de forma aproximada cuáles eran sus condiciones de trabajo y las principales necesidades a que debían hacer frente. Como ya el propio E. Iturriaga ponía de manifiesto, la mujer trabajadora se veía seriamente afectada por unas deficientes condiciones higiénicas fuera cual fuese su ámbito laboral. Por lo que se refiere al ámbito industrial, este autor considera que bien podrían extrapolarse -incrementadas seguramente, las cifras obtenidas en los EE.UU. por las que se constataba que la morbilidad de los operarios varones (101,1 por mil) era muy inferior a la de las hembras (154 por mil) (27). Esta situación sería atribuible a las malas posturas, al ambiente viciado y a las temperaturas extremas que tendrian que soportar las operarias en los talleres. En concreto, las enfermedades más habituales serían, según el citado estudio, la clorosis, la anemia y las propias de los órganos sexuales, cuyos negativos efectos sobre la capacidad reproductora no dejarían de ser subrayados por nuestro autor. En efecto, Iturriaga nos habla de una "natalidad" (28) (sic) del 4,8\% en las obreras industriales, frente al 10,97\% del conjunto de la población estadounidense; en Alemania, de 1.100 embarazos en obreras, só-

(26) ITURRIAGA, E.: “El trabajo y la mujer...», art. cit., pp. 391 ss.

(27) Cfr. Ibidem, p. 393.

(28) Se emplea aquí de forma impropia el término «natalidad». (relación entre los nacimientos y el total poblacional) para referirse a la "fecundidad" (relación entre los nacimientos y el colectivo de mujeres fértiles). 
lo se desarrollarían con normalidad $309(\simeq 28 \%$ ); si aceptamos las cifras del artículo, en España, durante estos años, la mortalidad infantil en hijos de obreras sería nada menos que del 175 por mil, frente al 114 por mil de la población total (29). Una vez más, los ideólogos del Régimen subordinaban la inserción de la mujer en el mundo del trabajo a la defensa de la actividad reproductora y de la maternidad, para ellos las funciones primordiales del colectivo femenino.

En íntima conexión con lo anterior habría que situar las demandas realizadas en 1938-39 por diversos higienistas sobre la necesidad de disponer de guarderías infantiles para los niños que quedaran "desatendidos», sanitaria y educativamente, al trabajar ambos padres fuera del hogar (30). Planteando esta exigencia en el caso de Castellón, J. Selfa nos revelará incluso en qué sectores industriales trabajaban las madres referidas, concretamente derivados agrícolas y géneros de punto (31).

Conocemos asimismo por el boletín, que la mujer trabajaba también en otras industrias. C. Bécares, en 1945 nos aporta un estudio sobre saturnismo industrial analizando 503 trabajadores, 25 de ellos mujeres, 2 de las cuales ostentaban la cualificación de fundidoras limadoras. Si bien este colectivo femenino se encontraba sólo levemente afectado por la intoxicación, una de las fundidoras limadoras había sufrido ya varios abortos espontáneos desde 1944 (32). En un trabajo sobre toxicidad industrial en Madrid se describe cómo en las industrias del caucho, en labores de máxima toxicidad (engomado de telas y otras piezas) el mayor porcentaje de puestos de trabajo está ocupado precisamente por mujeres (33). Aunque trabajando con datos referidos a 1928, Dantín Gallego destaca la participación de la mujer en la minería asturiana abordando tareas de exterior, a su juicio, con mayor eficacia que los hombres por su especial constitución física (34). Por último, aunque no exis-

(29) Cfr. Ibídem, p. 393.

(30) Entre otros, HERNÁNDEZ, I.: "La asistencia social al niño...», art. cit., p. 200.

(31) Cfr. SELFA, J.: "Información de los Servicios de Higiene infantil de Castellón durante los años 1936-37-38". RSHP, I, 1-12 (1938-39), pp. 272 s.

(32) Cfr. BECARES, C.: "Resultados y conclusiones de un estudio sobre saturnismo industrial». RSHP, XIX, 2 (1945), pp. 105-113.

(33) Cfr. QUINTANA, P. de la; DANTÍN GALLEGO, J.: "Estudio sanitario de algunas industrias que emplean disolventes». RSHP, XVIII, 4, (1944), p. 292.

(34) Cfr. DANTÍN GALLEGO, J.: "Antecedentes para el estudio de la Higiene...", art. cit., pp. 353 ss. 
ten estudios relativos específicamente a las condiciones de trabajo en el ámbito rural, conocemos algo de cuán precarias eran éstas a través de referencias indirectas como las mencionadas (convivencia con animales, permanencia durante el embarazo en el laboreo agrícola, etc.) (35).

No obstante, por lo que sabemos, cuando los higienistas del trabajo confeccionen fichas de seguimiento sanitario para los trabajadores, aun teniendo conocimiento de la peligrosidad que encierran determinadas tareas desempeñadas por mujeres, jamás recogerán en ellas datos que hagan alusión a las peculiaridades biológicas del sexo femenino (36).

Según datos del Anuario Estadístico publicado en el año 1942 y recogidos por la RSHP en 1943, la población de España en 1930 se situaba en torno a los 23,5 millones de personas (37), de los que más de 11 , el $51,36 \%$, serían mujeres. Sin embargo, su nivel de incorporación al trabajo sería extremadamente bajo, pues sólo el $9,12 \%$ de ellas formarían parte de la población activa real, constituyendo únicamente el $12,88 \%$ del total de ésta; los varones, por su parte, aun siendo menos de la mitad de la población, estarían incorporados al trabajo en un $65,13 \%$ y compondrían el $87,12 \%$ de la población activa real.

Por sectores, las mujeres se concentrarían fundamentalmente - según el citado Anuario- en el servicio doméstico (341.550), la agricultura (262.713), las industrias textiles (115.361), las confecciones $(90.415)$ y el clero $(60.170)$. Su número superaría ampliamente al de los varones en dos casos, el servicio doméstico (casi ocho veces) y las confecciones (más del doble); en el resto de las actividades citadas el número de mujeres rebasaría también, aunque en menor proporción, al de hombres, excepto, como es lógi$\mathrm{co}$, en el sector agrícola, donde se asentaría la mayor parte de la población activa masculina de la época. Por el contrario, las mujeres tendrían una exigua presencia en sectores como las "fuerzas públicas» (15), la metalurgia (128) o la minería (159), tradicionalmente privativos de los varones (38). También, según datos de

(35) Vid. supra, pp. 7-8.

(36) Vid. QUINTANA, P. de la; DANTÍN GALLEGO, J.: "Fichas para investigación sanitaria de industrias». RSHP, XVIII, 8 (1944), pp. 591-597.

(37) Concretamente 23.578.993, Cfr. QUINTANA, P. de la; DANTÍN GALLEGO, J.: "La Sanidad pública y la higiene en los lugares de trabajo». RSHP, XVII, 1-6 (1943), p. 605.

(38) Los datos se extraen de Ibídem, p. 607. 
1933 (39), en actividades concretas como, por ejemplo, la fabricación de conservas, la presencia de mujeres (19.607) sería sensiblemente superior a la de hombres (4.483). No obstante, la inmensa mayoría de la población femenina (más del $90 \%$ ) se encuadrarían abiertamente en la clase de los pasivos más de 9,3 millones de mujeres estarían, en efecto, catalogadas en 1930 sólo como "miembros de la familia", sin profesión declarada y sin desarrollar ningún tipo de actividad fuera de los márgenes del hogar (40).

En cuanto a la mortalidad profesional, las cifras que se emplean en la RSHP pertenecen a 1935 (41). Por lo que se deduce de ellas, el $93,43 \%$ de las muertes profesionales en mujeres se dan en el sector "diversas" (42), compuesto, entre otras actividades, por el trabajo doméstico y la prostitución. Si bien en otro momento hemos indicado que la morbilidad en las mujeres parecía ser superior a la observada en los hombres, debemos matizar ahora que esto no será así en lo que se refiere a la mortalidad; en efecto, en el grupo de las hembras, la tasa será del 14,72 por mil, mientras que entre los varones se elevará al 16,25 por mil. Por profesiones, la mortalidad será mayor entre las mujeres del grupo mencionado de "diversas" $(15,63$ por mil), seguido del de "rentistas y propietarias" (11,81 por mil) y del de las "profesiones liberales" (10,35 por mil); en los tres casos, casi la mitad de las tasas obtenidas en los grupos de varones. Por el contrario, la mortalidad será mínima entre las mujeres de la "administración pública» $(2,05$ por mil), el "transporte" (3,03 por mil) y la "explotación del suelo" (3,05 por mil), siempre con índices muy inferiores a los masculinos. También por debajo de éstos, pero en niveles ligeramente superiores a los últimos citados, estará la mortalidad femenina en la "industria» $(4,21$ por mil) y los «extractivos» $(4,14$ por mil).

Sin embargo, el trabajo femenino en el ámbito de la Sanidad y en el de la divulgación ideológica será el que más repetidamente se mencione en la RSHP por su propio carácter de órgano del apa-

(39) Cfr. DANTÍN GALLEGO, J.: "Antecedentes para el estudio de la Higiene...", art. cit., pp. 355 ss.

(40) Cfr. QUINTANA, P. de la; DANTÍN GALlEGO, J.: «La Sanidad pública y la higiene...n, art. cit., p. 610 .

(41) Cfr. VILLAR SALINAS, J.: "Mortalidades específicas profesionales en España". RSHP, XIX, 7 (1945), p. 459.

(42) Es significativo que el autor de esta clasificación -en contraste con lo que sería el criterio general- incluya entre las muertes "profesionales» a las de las "amas de casa" y otros sectores que él mismo llama "improductivos". 
rato sanitario del Régimen. Dos tipologías femeninas se verán continuamente ensalzadas como encarnación del nuevo papel social de la mujer española: la enfermera y la militante de la "Sección Femenina" de F.E.T. de las JONS.

De un lado, la profesión de enfermera se presenta como específicamente femenina. Dentro de la Sanidad, rara vez se le atribuirá mayor responsabilidad a la mujer, como se pone de manifiesto por la escasa participación que como teórica tiene en la revista. Se plantea, no obstante, una rigurosa selección de estas mujeres por su cualificación médica, psicofísica y moral, con el fin de elegir a la profesional «idónea» (43). Y ello será así porque en la postguerra las enfermeras "divulgadoras», "instructoras de Sanidad" o "visitadoras", constituirían la avanzadilla que supervisará directamente el cumplimiento de la normativa establecida por la Dirección General de Sanidad; entre otras labores, repartirían y recogerían los materiales sanitarios dispuestos para control y estudio estadístico de la situación socioeconómica e higiénica de la población, difundirían las medidas propuestas por el Estado para disminuir la morbimortalidad infantil y maternal e intentarían llenar el vacío de asistencia social y médica que existía en las zonas rurales (44).

Por otra parte, el "ejército femenino" (45), formado por las militantes de la "Sección Femenina" del nuevo partido único F.E.T. de la JONS, compartiría en buena parte con las enfermeras tanto las tareas sanitarias como las de divulgación ideológica, si bien su compromiso político sería más sólido. Su función era canalizar las inquietudes de las mujeres, intentando dar respuesta a sus carencias y necesidades desde los postulados del Régimen. Como exalta Iturriaga, la mejora de las condiciones de trabajo de las obreras habrían de venir de la mano de los Sindicatos dependientes de la "Sección" (46).

(43) Cfr. TUREGANO, J.F.; DANTÍN GALLEGO, J.; BENÍTEZ RAMÍREZ, A.: "Selección médico-psicotécnica...", art. cit., pp. 303 ss.

(44) Vid. SUÁREZ DE PUGA, L.: "Colaboración de las Divulgadoras rurales con la Sanidad nacional y el conocimiento y lucha contra la mortalidad infantil". RSHP (1941), pp. 397-400.

(45) MURILLO, F.: «Política de repoblación...», art. cit., p. 223.

(46) Cfr. ITURRIAGA, E. de: "El trabajo y la mujer...», art. cit., pp. 393 ss. 


\section{CONCLUSIONES}

De forma muy esquemática podríamos sintetizar así las principales conclusiones que pueden deducirse del trabajo:

1) La instauración del Régimen fascista de Franco supondrá la consolidación de un modelo por el cual el trabajo de la mujer tenderá a desvincularse del ámbito fabril, para desarrollarse fundamentalmente en el ámbito familiar, sobre la idea de que la principal función social de la mujer estaría en la reproducción y el adoctrinamiento de los hijos.

2) Pese a que en la legislación la mujer contara con derechos laborales similares a los de los hombres, lo cierto es que en la práctica su grado de incorporación al trabajo sería mínimo, afianzándose además la división de funciones en el trabajo por razón del sexo, lo que habría de dejar a mujeres relegadas a tareas secundarias en la producción, básicamente en el campo de la Sanidad y de la Asistencia social.

3) Asimismo, las condiciones de vida y de trabajo, tanto de las "amas de casa" como de las mujeres insertadas efectivamente en la producción, serían por lo general especialmente precarias, como se denota por la elevada morbilidad diferencial, haciéndose aún más negativas en las zonas rurales debido a la escasez de medios y de infraestructura.

4) Finalmente, a tenoe de lo visto, es posible entender la enorme dificultad que el colectivo femenino tendría para desarrollar una conciencia común, y más aún para avanzar por el camino de la autoconciencia obrera, precisamente por su fuerte desvinculación del ámbito fabril, convirtiéndose, por el contrario, a menudo en base social del Régimen, con un papel efectivo en la reproducción demográfica, social e ideológica del mismo. 